\title{
Study on Continuous Cropping Obstacle and Control Strategy of
}

\section{Medicinal Plants}

\author{
Xiuying Wang ${ }^{1, a}$ \\ ${ }^{1}$ College of Traditional Chinese Medicine, Jilin Agriculture Science and Technology College, Jilin City, Jilin \\ Province, 132101, China \\ aemail:
}

Keywords: Medicinal plants; Continuous cropping obstacle; Allelopathy; Ways of prevention and treatment

\begin{abstract}
After the continuous years of planting, the same species or closely related species in the same land even in the normal management situation will appear the phenomena, including growing worse, serious diseases and insect pests, yield reduction and quality deterioration, which are the continuous cropping obstacles. Based on the author's learning and practical experience, this paper firstly analyzed the hazards of continuous cropping obstacle, and then analyzed the causes of continuous cropping obstacles in medicinal plants, and finally put forward the prevention and control measures of continuous cropping obstacles in medicinal plants.
\end{abstract}

\section{Introduction}

With the increase of age or the cultivation of medicinal plants in continuous cropping, plant growth, yield and quality of medicinal materials are greatly reduced, such as rehmannia, ginseng, pseudo-ginseng, radix pseudostellariae, coptis and Chinese angelica, etc. In recent years, with the increasing demand for Chinese medicinal herbs, the area of continuous cropping or multiple cropping has become larger and larger, and continuous cropping obstacle has become a major problem restricting the development of Chinese traditional medicine in agricultural production. At present, the researches on crop continuous cropping are mainly concentrated in soybean and some protected vegetable. There is little research on the mechanism and cutting techniques of continuous cropping obstacles in medicinal plants. The allelopathic toxicity of plants is one of the most important factors for the continuous cropping obstacle of medicinal plants. This paper reviewed the continuous cropping obstacle of medicinal plants in order to provide reference for future research; It also solved the problems of continuous cropping obstacle and soil fertility decline in the process of medicinal plant production, and gradually changed the cultivation and production technology of traditional medicinal plants, and promoted the construction of GAP base and the development of traditional Chinese medicine in China.

\section{The harm of continuous cropping obstacles}

After experiencing the continuous years of planting, the same species or closely related species in the same land even in the normal management situation, there will be growing worse, serious diseases and insect pests, yield reduction and quality deterioration phenomenon, which is the continuous cropping obstacle. It was called continuous cropping barrier or continuous cropping obstacle in Japan. Europe and the United States called Replant disease or Replant problem; in China, 
we called continuous cropping problem. Whether it is food crops, oil crops, vegetables, medicinal plants or plantation and horticultural crops, there are different degrees of continuous cropping obstacles. The continuous cropping problem brought about the same plant diseases and insect pests, decrease of plant resistance, decrease of yield and quality and even severe plant death. Because ginseng and American ginseng are seriously avoid continuous cropping of medicinal plants, an average of more than $7000 \mathrm{hm}^{2}$ forests are logged annually in Jilin province so that to achieve rotation. Fusong, Jingyu, Ji'an and other old ginseng areas have basically no forest to cut; we return the grain plot to forestry when the trees were planted at the same time; however, because of single tree species, biodiversity loss and soil erosion have increased significantly; a series of ecological problems, such as reducing water resources, reducing soil fertility and unbalanced ecological balance of forests, have posed a serious threat to the ecological environment in Changbai Mountain.

Related reports show that seedling survival rate of continue cropping planting ginseng decreased to $30 \%$ in the second year; there are $70 \%$ of the ginseng burned off and overgrown with scars on root and skin. This resulted in the death of the upper part of the ginseng, and almost all of the plots were destroyed. Therefore, the problem of continuous cropping obstacle in old ginseng land has become a major technical problem that has been puzzling the development of ginseng industry. The growth environment of pseudo-ginseng is special and the planting area is limited with many serious continuous cropping obstacles. The most outstanding manifestation of pseudo-ginseng continuous cropping is "disease and low yield" problem. The average incidence of root rot in continuous cropping land was 23.9\%; it was equivalent to 3.5 times of that of new cultivated land. With the extension of continuous cropping years, the incidence of root rot disease was more serious. The continuous cropping showed that all the plants are died. Shorten rotation showed that the incidence of serious and survival rate is low, which resulted in low yield and poor quality. The most effective way to solve this problem is to cultivate new and non-host crops for more than 8 10 years. Rehmannia is a perennial herb and Rehmannia glutinosa is one of the famous "four big Huai medicine" in China. Both of them have serious continuous cropping obstacles, and the same ground interval can be replanted for 8 10 years, which seriously restricts the development of tunnel producing areas. In Huanghuaihai Plain honeysuckle planting GAP base, with continuous planting honeysuckle for more than 30 years, however in re-plantation, there are also many problems, including weak growth on new planting seedling, bad growth of roots, and short or small shoots, and the decreased flower (bud) amount. In the past 10 years, the rapid development of the agricultural production of Chinese medicinal herbs has made the problem of continuous cropping more and more prominent. Continuous cropping obstacle has become a widespread and serious production problem.

The reasons for the formation and aggravation of continuous cropping obstacles are complicated and various factors are interrelated and influenced to each other. Continuous cropping obstacle is the result of the combined effects of many factors in plant soil system, including soil infectious diseases, physical and chemical deterioration, allelopathic substances and so on. It has been thought that plant allelopathy is one of the main factors causing plant continuous cropping obstacle.

\section{Causes of Continuous Cropping Obstacle in Medicinal Plants}

Soil physical and chemical properties change. The studies show that the long-term continuous cropping of medicinal plants will lead to the destruction of soil aggregate structure, soil compaction, poor ventilation and deterioration of physical properties. For example, the study of ginseng shows that the proportion of soil and bulk density increases after planting; in addition, the 
pore of ginseng becomes smaller and the physical clay (less than $0.01 \mathrm{~mm}$ ) increases with decreasing the soil absorbing property and the soil acidification. However, some studies have shown that with the increase of continuous cropping years, the soil water stable aggregates increased from 0.25 to $2 \mathrm{~mm}$ and the soil permeability increased, but the soil physical properties improved.

Soil physical properties are influenced by many factors, such as climate, biology, topography, parent material and time, tillage and cultivation measures. Therefore, whether the continuous cropping of medicinal plants will cause the destruction of soil aggregate structure and the deterioration of physical properties need to be further studied. A large number of studies have shown that, because of the partial depletion of soil nutrients, continuous cropping will lead to uneven changes in soil nutrients, and thus directly affect the normal growth and development of the next crop. After continuous cropping of lily, the soil acidity increased and the organic matter lacked; moreover, the soil texture was sticky, and the soil available potassium was seriously deficient. The study of Salvia miltiorrhiza and aconite also found that with the increase of continuous cropping years, the soil tended to become acid, and the proportion of nutrient elements was out of balance. In addition, we found that soil organic matter, total nitrogen, total phosphorus and available phosphorus decreased with the increase of planting years, and soil phosphorus deficiency was serious through the soil fertility change study on perennial planting plants of Chrysanthemum, Salvia miltiorrhiza, Atractylodes macrocephala, Radix Isatidis.

Changes in soil biological environment. Continuous cropping of medicinal plants can change the biological environment of rhizosphere soil such as soil enzyme activity and soil microbial flora. With the increase of continuous cropping years, the microbial flora in the rhizosphere soil of Radix rehmanniae, Coptis chinensis and ginseng also changed significantly. The study of continuous Salvia miltiorrhiza showed that the acidic substances secreted by its roots affected the soil microbial community, especially the number of fungi. It can be seen that continuous cropping of medicinal plants has a great influence on soil enzyme activity and microbial flora in rhizosphere soil, which may be one of the main reasons for the continuous cropping obstacle. In addition, most medicinal plants have some exclusive pathogenic microorganisms and the years of continuous cropping can cause the accumulation of these pathogenic microorganisms. A vicious cycle of infection is harmful to medicinal plants, such as the beneficial microorganisms in the rhizosphere of ginseng, while pathogens and nematodes increase.

\section{Prevention and Cure Methods of Continuous Cropping Obstacles in Medicinal Plants}

Reasonable rotation. Rotation is an important measure to restore soil fertility, reduce diseases and insect pests, and alleviate the continuous cropping obstacles of medicinal plants. At present, the improvement of old ginseng land is mainly through the establishment of reasonable rotation system, such as water rotation, medicine rotation and grain selection, etc. Japan adopted the rotation of paddy fields, beans, wheat, maize, pasture, vegetables, flowers and fruit trees to shorten the rotation period of the ginseng from 30 60 years to 13 19 years; China also conducted ginseng rotation of Amorpha, alfalfa, asarum research etc, and then put forward 6 to 10 years of rotation. Studies have shown that American ginseng should rotate with perilla, and perilla seed can be used as fertilizer for American ginseng. In recent years, China has established a short-term rotation of each other technology system by utilizing a period of 1 3 years of ginseng and American ginseng. The technique of the new rotation system is feasible and practical, and the rotation effect is good.

Soil sterilization. At present, one of the important ways to overcome continuous cropping obstacles is the soil sterilization, especially for medicinal plant soil borne disease exacerbation of continuous cropping. Chemical fumigation is a commonly used method of soil sterilization. Methyl 
bromide (MBr) was once widely used as a soil fumigant for pseudo-ginseng continuous cropping. Because of its destructive effects on the atmospheric ozone layer, it has now been replaced by organic sulfur fumigant. Research shows that pseudo-ginseng continuous cropping used 98\% Dazomet powder agent(20 40 g/m $\mathrm{m}^{2}$ and 35\% potassium Wei fluid agent $\left(30 \sim 50 \mathrm{ml} / \mathrm{m}^{2}\right)$ before sowing and transplanting to make soil fumigation treatment of tillage layer. It had no significant effect on the rate of seedling emergence and seedling rate of pseudo-ginseng, but the amount of weed and nematode decreased by 90\% 95\% compared with the control. If the soil is fumigated and the probiotics can be added and combined with the use of high efficiency compound bactericide and the pseudo-ginseng continuous cropping obstacle can be solved well. In addition, disinfection of American ginseng with continuous cropping soil by matrix Steriland chloropicrin can significantly improve the survival rate and reduce the root disease.

Add beneficial microorganisms or nutrients. Supplementing and adding beneficial microorganisms or nutrients in the continuous cropping soil can improve the biotic and abiotic environment of the soil. It also can promote the growth and development of plants, inhibit the occurrence of diseases, improve the quality of medicinal herbs, and alleviate the continuous obstacle of medicinal plants to a certain extent. If beneficial microorganisms and nutrient solutions are added to the soil, the soil ring mirror of the old ginseng soil can be improved and ginseng pathogenic bacteria can be suppressed. Continuous cropping obstacle of medicinal plants is often the result of synergistic effects of many factors. It is difficult to achieve good governance effect by adopting the above single prevention and cure measures. At present, the comprehensive measures such as physical measures, chemical measures and agronomic measures are adopted to deal with the continuous cropping soil; good results have been achieved to alleviate the continuous cropping obstacles of the old ginseng land.

\section{References}

[1] Hou Hui, Dong Kun, Yang Zhixian, Dong Yan, Tang Li, Zheng Yi. Advances in Research on Mechanism of Continuous Cropping Obstacle[J]. Soil, 2016, (06): 1068-1076.

[2] Zhuang Yue'e, Chen Huaguan. Research Progress of Continuous Cropping Obstacle and Molecular Ecology Mechanism of Medicinal Plants[J]. Strait pharmacy, 2015, (11): 4-8.

[3] Tan Guoyin, Yang Zhiling, Yuan Zhilin, Yang Xu. Research Progress of Continuous Cropping Obstacles and Control Approaches of Medicinal Plants[J]. Journal of Northwest Agriculture and Forestry University (Natural Science Edition), 2012, (04): 197-204.

[4] Liang Zhihuai, Wei Lin, Li Shidong, Li Jiguang. Advances in Studies on Plant Pathogenic Continuous Cropping Disorder[J]. Hunan Agricultural Science, 2012, (05): 73-77.

[5] Zhang Min, Tan Xianhe, Zhang Yu, Huang Xiaofen. Continuous Cropping Obstacle of Chinese Medicinal Herbs[J]. Bulletin of Agronomy of China, 2009, (23): 142-146.

[6] Li Xueli, Lee Jung, Li Yantao, Zhang Wenping, Ceng Xianli, Zheng Wenran, Liu Guoshun, Ye Xiefeng. Advances in Allelopathy of Plants[J]. Bulletin of Agronomy of China, 2009, (23): 142-146. [7] Zhang Zhongyi, Lin Wenxiong. Allelopathic Toxicity and Continuous Cropping Obstacle of Medicinal Plants[J]. Chinese Journal of Ecological Agriculture, 2009, (01): 189-196. 